\title{
Anti-PSMA/CD3 BiTE Monoclonal Antibody MT112
}

National Cancer Institute

\section{Source}

National Cancer Institute. Anti-PSMA/CD3 BITE Monoclonal Antibody MT112. NCI

Thesaurus. Code C103827.

A recombinant $\mathrm{T}$-cell eng aging bispecific monoclonal antibody (BITE) directed against human prostate specific membrane antigen (PSMA) and the CD3 epsilon subunit of the T cell receptor complex, with potential immunostimulating and antineoplastic activities. Anti-PSMA/CD3 BiT E monoclonal antibody MT112 possesses two antigen-recognition sites, one for PSMA, and one for the CD3 complex, a group of T cell surface glycoproteins that complex with the T cell receptor (TCR). This bispecific monoclonal antibody brings PSMA-expressing tumor cells and cytotoxic T lymphocytes (CTLS) together, which may result in the CT L-mediated cell death of PSMA-expressing cells. PSMA, a tumor associated antigen, is overexpressed on the surface of metastatic and hormone-refractory prostate cancer cells. 